\title{
Demokratie - zwischen Kampfbegriff und Nebelkerze. Was können Sprach-, Medien- und Kulturwissenschaften zur Demokratisierung von Gesellschaft beitragen?
}

\author{
Clemens Knobloch (Siegen) und Friedemann Vogel (Freiburg)
}

\begin{abstract}
Everybody speaks about democracy. In current media discourses, 'democracy' means everything and nothing. You can explain war as "democratization" (of Iraq, Afghanistan etc.) just as well as you can call a referendum about fundamental political decisions "terrorism" (like in Greece 2015). Is democracy no more than a buzzword? This contribution explores the relationship between language and democracy both in a descriptive and a normative perspective. What can linguistics, media and communication science contribute to this topic? How does the public spectacle of democracy work? And what is the notion of "democratic speech"?
\end{abstract}

\section{Sprache und Demokratie: ein Annäherungsversuch}

Sprachen, so scheint es, sind zutiefst demokratische Institutionen. Sie existieren (um eine Formulierung des sprachkritischen Fritz Mauthner zu verwenden) nur "zwischen" den Menschen. Sie gehören allen. Ihre Ressourcen können weder privat angeeignet noch von mächtigen Gruppen für sich monopolisiert werden. Sie stehen allen zur Verfügung, die sich ihrer zu bedienen wissen. Während andere Güter durch vielfältigen Gebrauch abnehmen, werden Sprachen dadurch reicher. Selbst hermetisch formulierte Äußerungen bleiben unumgänglich offene Sinnangebote bei ihrem Wandel durch die Kontexte.

Und doch täuscht der demokratische Schein. Denn wie alle Institutionen sind auch Sprachen nur insoweit potentiell demokratisch, wie sie von sozialen Wesen, mündigen Sprecherinnen und Sprechern, mit Leben gefüllt werden. Da entstehen alsbald Zweifel: Ist nicht jedes sprachlich formulierte Sinnangebot, da es doch das Bewusstsein des Rezipienten "steuern" muss, um verstanden zu werden, zugleich "Manipulation" (wie man oft hört)? Auf "die Medien" wird gerne verwiesen, die das Deutungsgeschäft für sich monopolisiert hätten und uns mit handlichen und plausiblen Schablonen versorgen in all den Bereichen, von denen wir keine unmittelbare Erfahrung haben (und das sind so gut wie alle!). Und selbst unsere höchst persönlichen Angelegenheiten müssen, wenn sie (uns) öffentlich kommuniziert werden, durch das Raster der medialen Sagbarkeit. 
Sagbarkeit: Demokratie heißt, bei den zentralen Entscheidungen des sozialen Zusammenlebens partizipieren, (mehr als nur) ein Wörtchen mitreden zu können. Was aber, wenn einem die Sprache versagt? Wenn Partizipation an der Sagbarkeit scheitert? Nehmen wir den Asylsuchenden ("Asylbewerber"), der bei seiner "Anhörung" vor deutsch-fremden Beamten von Vergewaltigung berichten soll, in fremden oder nur vermittelten (weil übersetzten) Worten, in einem Modus, der nicht der seine ist, im Kontext einer (Verwaltungs-)Sprache, die selbst den meisten Muttersprachlern ungeläufig ist. Und über seiner Aussage, im besten Fall geübtfreundlich honoriert, schweben mit der Drohung sofortiger Abschiebung auch Verfolgung und Tod. Oder nehmen wir den umgekehrten, nicht nur in Guantanamo, sondern auch in Deutschland 2002-2004 prominent gewordenen Fall des (angedrohten) Folterverhörs (Metzler-Entführung und Daschner-Prozess zur sog. "Rettungsfolter"), in dem das Recht zu Schweigen gebrochen und eine fremde als die eigene Stimme erpresst wird. Auch Fragen der Zensur, des Sprechen-Dürfens, und der Selbstzensur, des freien Sprechen-Könnens, sind nicht Phänomene unter ferner liefen, sondern gehören auch hierzulande zur sprachlichkommunikativen Ethnographie der politischen Alltagskommunikation.

Versteht man Demokratie als die Voraussetzung, Menschenrechte und also individuelle Menschenbedürfnisse gewaltfrei und gleichberechtigt im Kollektiv aushandeln zu können (vgl. Narr 2012), bedarf es aber noch mehr. Zumindest mehr als nur die allvierjährliche "Wahl" zwischen rhetorischen Formeln oder alternativ dem falschen Satz, es könnten nicht immer alle überall mitreden (denn meist zählt der Sprecher dieses Satzes nicht zu diesen "allen"). Denn das Mitreden, das zeigen Formate wie die Wikipedia, lässt sich durchaus organisieren: Digitale Ressourcen wie das Internet müssen nicht notwendig nur für Privatwirtschaft und Imagepflege missbraucht werden (vgl. Rilling 1998), sondern können unter bestimmten Bedingungen auch neue Kommunikations- und Partizipationsmöglichkeiten schaffen (zur Diskussion vgl. Coleman 2007). Dass damit Schwierigkeiten verbunden sind, versteht sich von selbst. Was können Sprach- und Kommunikationswissenschaftler zur Lösung dieser Probleme beitragen, wie können sie Partizipation am Sprachspiel befördern, notwendig als Mitspieler und nicht nur als (gar öffentlich orakelnder) Kommentator neben dem Spielfeld?

Nötig wäre Partizipation auch gerade dort, wo sie vermeintlich medial zu Hause ist, nämlich im "Gesellschaftsspiel" des öffentlichen Sprechens. Dort freut sich der einzelne, wenn er die von Medien veröffentlichten, zusehends professionell produzierten Formeln der gesellschaftlichen Selbstdeutung nach- und mitsprechen kann, von der "Globalisierung" mit ihren Sachzwängen über die von multiplen "Krisen" bedrohlich umstellten Horizonte bis zum geheimdienstlich wegbereiteten "Krieg gegen den Terror". Selbst die "Postdemokratie"-Diagnose wird gedanken- und folgenlos mitgesprochen. Die politischen Sprecher dieser Sprache schaffen erst, was sie zu diagnostizieren vorgeben, und damit zugleich den Humus ihrer Handlungsinteressen. Da wird Sprache zum hegemonialen Transmissionsriemen, zum Schmiermittel tendenziösen Reframings (vgl. Goffman 1974): Wie kodiert man etwa rapide wachsende soziale Ungleichheit auf "Bildung" um (Armut heißt jetzt "bildungsferne Schichten")? Wie definiert man Verfassungsrecht auf soziale und kulturelle Teilhabe um zu einer lebenslangen Selbstoptimierungspflicht im Interesse des Arbeitsmarktes? Wie verschanzt man Interessenpolitik zugunsten des Finanzkapitals hinter einwandsimmunen und zustimmungspflichtigen Fahnenwörtern wie "Wettbewerbsfähigkeit" und "für die Freiheit"? Wie lernt man es, 
"Demokratie" - zwischen Kampfbegriff und Nebelkerze

Ereignisse und Handlungen möglichst automatisch so zu kodieren, dass das Transkript dem eigenen Image entspricht? Wie erzeugt man öffentlich Ängste (z. B. vor gesellschaftlicher Überalterung und Altersarmut), die dann von interessierten Anbietern privater Dienstleistungen aufgegriffen werden können (z. B. private Altersvorsorge)? Wie bringt man ein Publikum dazu, sinkende Standards der öffentlichen Daseinsvorsorge (in Bildung, Gesundheit, öffentlichem Verkehr usw.) ohne Protest hinzunehmen? Könnte man flächendeckend öffentliche sonderpädagogische Einrichtungen schließen und abbauen, wenn man nicht das Zauberwort "Inklusion" dafür hätte, das jenseits fachdidaktischer Diskussion den unschätzbaren Vorteil bietet, jeden, der sich dagegen wehrt, als Befürworter der "Exklusion" von Behinderten dastehen zu lassen? Und gibt es auch andere Möglichkeiten, ein ziemlich egalitär aufgestelltes und meritokratisches öffentliches Hochschulsystem reputativ zu spreizen und für Privatisierung zu öffnen, als durch das Versprechen von "Exzellenz" und "Elite" am oberen und "Beschäftigungsfähigkeit" am unteren Ende des Spektrums?

Unmerklich haben sich Regeln und Maximen der Machtkommunikation etabliert, an denen sich die Akteure ebenso orientieren wie die Erwartungen des massenmedialen Publikums (zur Mediokratie vgl. Meyer 2002). Jeder ahnt, dass in der Internet- und Handykommunikation heute nichts mehr privat bleibt. Dann stellt sich heraus, dass US-amerikanische und europäische Geheimdienste milliardenfach Privatdaten anzapfen. Wenn die Medienöffentlichkeit für die Dauer eines Features darüber debattiert, geben die Geheimdienste eine unkonkrete, doch medial multiplizierte Terrorwarnung an die Bevölkerung aus (so Anfang August 2013 nach neuen Vorwürfen gegenüber Geheimdienstüberwachung oder auch zuletzt wieder im Mai 2015 nach Bekanntwerden der Kooperation von Bundesregierung, Bundesnachrichtendienst und US-Geheimdiensten beim Ausspähen europäischer Politik und Wirtschaft). Medienöffentliche Debatten folgen den aufmerksamkeits- und profitökonomischen Potentialen von Ereignissen (Events) und Handlungen. Oder sie inszenieren Ereignisse nach diesen Potentialen. Ihr Zeithorizont für Ereignisse ist kurz, ihr Gedächtnis notorisch schwach. Einen sehr langen Atem haben sie dagegen bei der Etablierung konnotativer Zusammenhänge, bei der Rahmung komplexer Sachverhalte durch einfache, wiederkehrende Deutungsfiguren. Die Logik ist okkasionalistisch: Gelegenheiten müssen genutzt, besser noch: erzeugt werden. Die öffentliche Aufmerksamkeit ist eine knappe Ressource, und sie wird penibel bewirtschaftet. Wer untersucht die pragmatischen Regeln der Meinungsbildung im öffentlichen Diskurs, nach denen nur dasjenige zum Skandal werden kann, was jeder eigentlich schon weiß und sich daher in die rekurrenten Deutungsmuster einfügt? Wer verfolgt die Fach- und Praxisdiskurse, in denen offen verhandelt wird, wie öffentliche Meinung und politische Zustimmung so planmäßig hergestellt werden können wie Waschmittel und Autos?

\section{2 "Demokratie" im öffentlichen Diskurs}

$\mathrm{Zu}$ den wiederkehrenden, einfach schematisierenden Deutungsfiguren gehört bedauerlicherweise auch die Art und Weise, wie in den letzten Jahrzenten das Konzept Demokratie in den öffentlichen Diskursen verarbeitet wird. ${ }^{1}$ Kurz gesagt: "Demokratie" sind präsuppositionsreich und unkonkret wir, die westlichen Guten, weil - und nur weil - alle

\footnotetext{
1 Zum historischen Demokratiediskurs vor und nach 1945 sowie im Kontext der 60er/70er Jahre vgl. Kilian 1997, 2003 sowie Kämper 2009, 2012, 2013; Kämper, Haslinger und Raithel 2014.
} 
anderen das negative Gegenstück bilden. Zu diesem Schluss jedenfalls kann schon kommen, wer den Gebrauchskotext von Demokratie im Deutschen Referenzkorpus (DeReko) des Instituts für Deutsche Sprache kursorisch betrachtet. Das DeReko enthält derzeit über 8 Milliarden fortlaufende Wortformen, von denen ein Großteil Medientexte (aktuelle Zeitungen, Zeitschriften), aber auch etwa die Diskussionsseiten der deutschsprachigen Wikipedia sind. Die 236.119 von uns erfassten Belege ${ }^{2}$ haben wir mit Hilfe einer statistischen Kookkurrenzanalyse (mittels Cosmas www.cosmas2.ids-mannheim.de, [24.09.2014]) strukturiert, nach wiederkehrenden Kotextmustern (repräsentiert in Form von Kookkurrenzpartern, KKP) sortiert und die ersten 100 hochsignifikanten Kotextmuster mit Hilfe von Volltextanalysen der dazugehörigen Belege ausgewertet. ${ }^{3}$ Induktiv nach Gebrauchsähnlichkeit gruppiert verdichten wir die Ergebnisse wie folgt:

a) Grundsätzlich wird Demokratie vor allem in drei verschiedenen Modi konkretisiert: 1) gar nicht (d. h. hochgradig implikativ), 2) selten positiv (v. a. als parlamentarische / repräsentative, durch Wahlen legitimierte, westliche gesellschaftliche Organisationsform) als ein Element einer sich gegenseitig nur äußert abstrakt (und insofern wiederum hoch implikativ) konkretisierenden Aufzählung wiederkehrender Hochwertwörter oder 3) über Negationen.

b) Konkretisierung mittels Aufzählung: Demokratie gilt oft in einer FundamentMetaphorik als Grundsatz, Grundwert, Grundelement, Grundpfeiler o. ä., als indiskutables (allgemeingültiges, unverzichtbares, universelles) Prinzip, dessen Werte zu erhalten seien. Diese wiederum höchst abstrakten Werte einer als gemeinsamen (d. h. vor allem unsere / europäisch / westlich homogenisierten) Wertegemeinschaft werden dann flexibel eingesetzt oder - gemeinsam mit Demokratie - in eine parallelisierende Reihe gesetzt. Wiederkehrend in unterschiedlichsten Konstellationen und verschieden umfangreich genannt werden:

Freiheit/freiheitlich, Frieden, Menschenrechte, Rechtsstaat, Toleranz, Gerechtigkeit, Presse[freiheit], politische Willensbildung [-> Wahlen], Wahlen, Meinungsfreiheit, Informationsfreiheit, Rechtsstaat, Gewaltenteilung, Ehrenamt, Transparenz, Marktwirtschaft, Parlamentarismus, Pluralismus.

Der (Sprecher-)inkludierende Wertekatalog dient quasi nie dem Versuch, Demokratie positiv(istisch) zu verhandeln. Vielmehr wird ein konkreter erwünschter oder unerwünschter Sachverhalt den dafür jeweils eigens zusammengestellten Katalogen gegenübergestellt und rhetorisch bewertet. Die Aufzählungen dienen dann schnell als Topos zur Verpflichtung von Dritten zu X (feste Wendung: für Werte der Demokratie eintreten) oder zur Exklusion von Unerwünschten, zum Beispiel Migranten oder EU-interessierten Fremdstaaten.

wir [können] sehr wohl von allen Zuwanderern erwarten: Dass sie sich in zentralen Punkten für die Werte unserer Demokratie öffnen. Das ist keine unzumutbare Hürde, das ist eine schlichte Selbstverständlichkeit.

(BRZ06/FEB.07389 Braunschw. Z., 14.02.2006)

\footnotetext{
$299 \%$ der Belege entstammen aus den 90-00er Jahren; unter $1 \%$ (aber immerhin noch rund 1.300 Belege) stammen aus den 70er und v. a. 80er Jahren.

3 Damit vereinfachen wir (im doppelten Sinne) die Analyse erheblich und vernachlässigen tendenziell niederfrequente Konstruktionen, können aber zuverlässige Aussagen über das Groß der semantischen Variation im öffentlichen Gebrauch (der 90er und 2000er Jahre) des Ausdrucks treffen.
} 
"Demokratie" - zwischen Kampfbegriff und Nebelkerze

Dieses "Pudding"-Fundament wird nicht in Frage gestellt (sondern soll - so die wiederholte Forderung - durch plebiszitäre Elemente ergänzt / gestärkt werden), im Gegenteil: mit einem Bekenntnis zur Demokratie soll häufig etwas, das als abzulehnen präsupponiert wird, "abgeschworen" (z. B. einer vorwurfsanfälligen SED-Mitgliedschaft) oder eine Gruppeninklusion vollzogen werden:

Als ehemaliges Mitglied der SED, das offen mit seiner Biografie umgeht, halte ich das Bekenntnis zu Demokratie und Freiheitsrechten für unverzichtbar, um glaubwürdig für eine freie, solidarische, eine lebenswerte Welt [... eintreten zu können].

(Wikipedia, Ost[...] 21:15, 06.06.2009 (CEST))

c) Konkretisierung durch Negation: Die häufigste Form zur Konkretisierung von Demokratie ist indirekt zu sagen, was "ihr" fehlt (mangelt / fehlt) oder was sie nicht sei. Was fehlt Demokratie? Stärke, Lebendigkeit, Direktheit, Echtheit. Diese Perspektive verfolgen zum einen jene, die die aktuelle gesellschaftliche Ordnungsstruktur (oder Teile davon) ablehnen und fahnensyntaktisch mehr "Partizipation von unten" gegenüber repräsentativen, lobbyistischen usw. Entscheidungsprozessen (Männerbünden, politischen Filz, Scheindemokraten, Marktradikale usw.) einfordern. [Mehr] Direkte Demokratie als etabliertes Fahnensyntagma und usuelle Wortverbindungen wie echte Demokratie (gg. Scheindemokratie, Pseudodemokratie) zeigen den Versuch, den Ausdruck Demokratie in seiner schillernden Polysemie zu markieren und referenzseitig neu zu besetzen. In ihrem näheren Kotext finden sich allerdings häufig die gleichen Hochwertworte (ohne nähere Konkretisierung) wie bei Nichtkritikern.

Ebenfalls eine "Mangel"-Konkretisierung lässt sich über den verbalen Kookkurrenzpartner leben (gelebte Demokratie, Demokratie muss leben durch X, Demokratie lebt von X u. ä.) verfolgen. Die Kritik stellt jedoch nicht die Systemfrage (wie oben tendenziell), sondern dient als Topos zur Verpflichtung der Bürgerinnen und Bürger 1) zur (Urnen-)Wahl, 2) für ehrenamtliches Engagement, 3) zur "konstruktiven Mitsprache oder zum Schweigen" (anstelle von Mitentscheidung: Bringen Sie sich ein, tragen Sie Ideen und Anliegen an uns heran; Demokratie lebt vom Mitmachen [und nicht vom Zuschauen / Meckern / Mitmeckern / Rückzug ins Private]). Mangelnde Wahlbeteiligung wird dann zur Verdrossenheit und zur Bringschuld der Verdrossenen:

Demokratie kann man aber nicht konsumieren, man muss sie aktiv leben. Die Verdrossenen sind an ihrer Verdrossenheit selbst mehr schuld als die Politik.

(Franz Müntefering 2008, zitiert nach Die Zeit vom 16.01.2008)

\section{Was ist Demokratie nicht oder genauer, wer hat sie nicht?}

- Rassisten, Rechtsradikale, (oder pauschaler) jede Form von Extremismus: gegen Fremdenfeindlichkeit / Intoleranz / Rechtsextremismus / Antisemitismus / Extremismus / Gewalt, Feinde der Demokratie (v. a. zur Realisierung des Topos: "Wenn X gegen die Demokratie ist, hat X kein Recht auf demokratische Rechte"4); ihnen gegenüber tritt die wehrhafte Demokratie, die - schon früher als Teil der verfassungsrechtlichen Universalkeule "Frei-

\footnotetext{
${ }^{4}$ Typisch zum Beispiel: Die Demokratie finanziert ihre Feinde [=NPD], die nichts anderes im Sinn haben, als die Demokratie abzuschaffen.
}

ISSN 1615-3014 
heitlich-demokratische Grundordnung" (FdGO) - heute nahezu jede Form präventiver staatlicher Gewalt und Überwachung zu legitimieren sucht.

- Die Diktatur des Proletariats; all jene Länder oder Regionen, die Demokratie nach westlichem [Zuschnitt / Muster / Vorstellung] nicht verfolgen, deren Demokratie nur halbwegs funktioniert und die Nachhilfe in Sachen/Punkto Demokratie, auf dem [steinigen / schwierigen / schmerzhaften = 'Opfer notwendig machenden'] Weg zur Demokratie benötigen. Es handelt sich vor allem um asiatische oder slawische, ehemals oder auch heute noch als 'kommunistisch' oder 'islamisch/islamistisch' akzentuierte Länder; allen voran: Russland, China, Afghanistan, Libyen, Türkei, Pakistan, Asien, Kuba, Serbien, Irak, Tunesien, Nigeria, Spanien [unter Franco], Ägypten, Ostblock, Afrika, Hindukusch, Jugoslawien. Das 'Problem' sind zum Beispiel gelenkte Demokratie (als Stigmasyntagma für Russland), Bauernvolk, Stammeskultur, kein Mehrparteiensystem, mangelnde Stabilität, [Israel inmitten] arabisch-islamischer Diktaturen oder [mangelnde] Marktwirtschaft.

jene Länder stärker als bisher zu unterstützen, die Marktwirtschaft und Demokratie weiterzuentwickeln (N91/AUG.08754 Salzburger Nachr., 21.08.1991) / Die anschließende Aufgabe, seine Heimat zu Demokratie und Marktwirtschaft zu führen, erwies sich als heikel (N93/SEP.33477 Salzburger Nachr., 15.09.1993) / gemeinsamer Interessen und des gemeinsamen Wunsches, daß aus Rußland eine Demokratie wird, die die Marktwirtschaft praktiziert (I96/JAN.00629 Tiroler Tagesztg., 08.01.1996).

Positiv wird die westliche Demokratie - soweit wir sehen - kein einziges Mal konkretisiert. Das Syntagma dient - genauso wie etwa die Bigramme Demokratie festigen, liberale Demo$k_{\text {ratie }}^{5}$ oder moderne Demokratie 6 - meist allein zur pejorativen Markierung und Exklusion von Fremdstaaten oder damit verbundenen Attributen.

d) Der statistisch häufigste Akteur im Kontext von Demokratie ist erwartungsgemäß das als globale Einheit präsupponierte Subjekt Volk, nach dessen Wille (als Souverän) Entscheidungen getroffen werden sollten. Über das Volk erfährt man im Kontext in der Regel nichts, es sei denn es ist (vor allem in Fremdstaaten) [noch nicht] reif für die Demokratie oder es ist Teil von deontisch bewerteten Handlungen (das Volk [soll / muss / will [wieder / erstmals usw.] frei wählen / bestimmen usw. können). Mit anderen Worten: der Souverän, also die Basis der Demokratie ist in unseren Belegen in der Regel eine unkonkretisierte Chimäre, der die Meinung des jeweiligen Autors in den Kollektivmund gelegt wird. Sehr viel konkreter sind hingegen unzählige onomastische Muster, d. h. Namen für zivilgesellschaftliche Organisationen, Vereine, soziale Bewegungen usw., die im Namen einen "partizipatorischen Mangel" implizieren und sich für die Beseitigung dieses Mangels (für Demokratie) einsetzen. ${ }^{7}$ Als Ak-

\footnotetext{
${ }^{5}$ Vgl.: liberale Demokratie, die sich noch immer als "Leuchtturm der Nationen" versteht.

6 "Heiligkeit des Gesetzes" zu reden? Eine solche sollte auch in der modernen Demokratie existieren / Spagat zwischen Islam und moderner Demokratie / seinen früheren Äußerungen zum Trotz, für eine Synthese aus muslimischer Tradition und moderner Demokratie zu stehen, auch Erdogan will Ost nicht einfach durch West ersetzen / Außerdem seh ich nicht ein, England mit einer modernen Demokratie zu vergleichen. / Zwischen Tradition und Moderne hat die Demokratie in der jungen Republik Usbekistan noch einen schweren Stand / Sie will ein Ende des Gottesstaates, eine moderne Demokratie, die Trennung von Staat und Religion u. a.

7 Omnibus für Direkte Demokratie, Volksinitiative "für Beschleunigung der direkten Demokratie" (Denner Initiative) [Schweiz], Organisation für direkte Demokratie durch Volksabstimmung, Bündnis für Demokratie und Toleranz, Jugend für Toleranz und Demokratie [Bundesprogramm], Vereinigten Front für Demokratie gegen Diktatur (UDD) [Thailand], Volksallianz für Demokratie (PAD) [Thailand, gilt als nationalistischrevanchistisch], prokurdische Partei für Frieden und Demokratie (BDP), Demokratie jetzt, Tibetischen Zentrums
} 
"Demokratie" - zwischen Kampfbegriff und Nebelkerze

teure (manchmal auch als Orte konzeptualisiert) fallen ferner wiederkehrende Fremdstaaten auf, die im Zusammenhang mit dem Ausdruck Demokratie in aller Regel negativ bewertet werden (vgl. oben c). Der mündige Bürger ist im Kontext solcher Länder selten oder gar nicht vermerkt, sondern stets im deutschen oder zumindest westlichen Raum anzutreffen. Er hat häufig mangelndes Vertrauen (in X), ist Teil der wehrhaften Demokratie und wünscht sich als Souverän [mehr] Partizipation, Teilhabe, mit- (Mitgestaltung, mitdenken, mitlenken, mitspielen, Mitwirkung, Mitbestimmung), Beteiligung usw. Sein Engagement ist Voraussetzung für eine "gelingende" Demokratie. In aller Regel inkludiert der mündige Bürger den Autor und generalisiert passivierend dessen Einstellung oder Forderung:

Mündige Bürger in einer Demokratie lassen sich von solchen Drohungen nicht beeinflussen (O00/JAN.10331 Neue Kronen-Ztg., 26.01.2000). / In einer Demokratie dürfen mündige Bürger auch Gerichtsurteile kritisieren (RHZ13/OKT.06356 RZ, 07.10.2013). / Schließlich gehören mündige Bürger zu den Eckpfeilern der Demokratie. An einer Meinung wider besseres Wissen festzuhalten, nur weil sie so schön in ein Schema passt [...] (M11/NOV.02867 Mannh. Morgen, 09.11. 2011).

e) Demokratie in Deutschland ist - von der oben skizzierten Mangelakzentuierung - rein positiv konnotiert, der implizite Sehepunkt für die jeweilige Bewertung von "undemokratischen" (d. h. also: nicht-westlichen, nicht-christlichen, nicht-marktwirtschaftlichen) Fremdsystemen. "Die deutsche Einheit ist ein Sieg von Demokratie und Menschenrechten", erklärte Scharping (M95/509.21769). Deutschland ist eine gefestigte Demokratie. Und Die Reformen haben hier gegriffen. Deutschland ist derzeit wahrscheinlich die stabilste Demokratie Europas (gemeint sind hier: stabile Marktverhältnisse, gesellschaftliche Ruhe u. ä.). Der von Willy Brandt in seiner Regierungserklärung 1969 geprägt Slogan Mehr Demokratie wagen ${ }^{8}$ ist das häufigste Zitat in Sachen öffentliche Demokratie-Rede, gefolgt von Wir brauchen Demokratie wie die Luft zum Atmen (Gorbatschow); nur vereinzelt Demokratie ist nicht alles, aber Sozialismus ohne Demokratie ist nichts (Bebel). Um den gefeierten Titel Wiege/Geburt der deutschen Demokratie konkurrieren vier unterschiedliche Daten miteinander: 1832 (Hambacher Fest) versus 1848 (Paulskirche / dt. Nationalversammlung) versus 1918 (Novemberrevolution) versus 1948 (Parlamentarischer Rat).

Unter den über 230.000 Belegen des DeReko finden sich lediglich 951 Treffer, die mit den Kotextausdrücken bedeuten oder heißen auf eine metasprachliche Konkretisierung und (via adversativer und konzessiver Konnektoren) eine Diskussion von Attributen verweisen. Eine Auswertung dieser Belege (mit Hilfe von Kookkurrenzanalysen 2. und 3. Ordnung $[+2 /+10]$, Konkordanzen und Volltext) zeigen folgende Konzeptbündel:

- (Abstrakte) Handlungsrechte: Wahlen / Wahl / Wahlrecht (Wahl zu haben zwischen politischen Alternativen); freie Meinungsäußerung; mehr als nur [das Recht auf freie Meinungsäußerung / ab und zu wählen zu dürfen]; Recht(e); Menschenrechte.

\footnotetext{
für Menschenrechte und Demokratie, Die Partei Regenbogen Äthiopien: Bewegung für Demokratie und Soziale Gerechtigkeit, Archiv der sozialen Demokratie [FES], UDPS (Union für Demokratie und Sozialen Fortschritt [Kongo, Partei], Partei der Sozialen Demokratie [Rumänien], Komitees für Grundrechte und Demokratie, Komitee für Demokratie und Sozialismus [Kuba] und andere Komitees, Echte Demokratie jetzt! und viele andere. Wir vermuten, dass mit diesen abstrakten "Pro-Demokratie"-Initiativen konkrete Interessen verfolgt werden, z. B. Kritik an lokal- oder regionalpolitischen Entscheidungsstrukturen.

${ }^{8}$ Von Angela Merkel in ihrer Regierungserklärung 2005 zu mehr Freiheit wagen variiert.
} 
- Deontisch (d. h. als wünschenswert) bewertete Handlungen: sich in die eigenen Angelegenheiten einzumischen. [Max Frisch, häufiges Zitat]; Meinungen [zulassen / akzeptieren]; Kompromiss; alle vier Jahre zur Wahlurne gehen/schreiten [reiche nicht aus]; entscheiden; akzeptieren; einmischen; gemeinsame Lösungen suchen; sich Mehrheitsentscheidungen zu beugen / Mehrheitsentscheidungen akzeptieren; streiten; [Ausnahme] Demokratie heißt: Man darf sich aussuchen, von wem man sich verarschen läßt.; Mitmachen; Mitbestimmung / Beteiligung; [X des / dem] anderen akzeptieren / zuhören I tolerieren; miteinander reden; auch Arbeit.

- Deontisch (d. h. als wünschenswert) bewertete Attribute oder Haltungen von Akteuren: Verantwortung; Fähigkeit zum Kompromiss; Toleranz; Wille des Volkes; gegenseitigen Respekt.

- Struktur- oder Prozessprinzipen: nicht Herrschaft / nicht Diktatur der Mehrheit; dass die Mehrheit entscheidet; Volksherrschaft / Herrschaft / [alle] Macht des Volkes; Entscheidung durch die Betroffenen; Macht auf Zeit; Ordnung durch Verfahren; Gegensatz, zur Diktatur; Legitimität durch Wahlen; Streit; Diskussion; Vielfalt der Kulturen; Wettbewerb um bessere Lösungen u. ä.; Mehrheitsprinzip.

- Akteure: Menschen; Bürgerinnen und Bürger; starke Opposition; Minderheit (Schutz I Toleranz der Minderheit, Minderheit bald Mehrheit, Unzufriedenheit der Minderheit); Mehrheiten [gewinnen / akzeptieren / hören / organisieren]; Regierung; Bevölkerung; Betroffene / diejenigen, die von einer Entscheidung betroffen sind; Parteien; [X des] anderen; Andersdenkende; Volksvertreter.

- Orte: Parlament.

Die hier ausdrucksseitig zusammengetragenen Sprachgebrauchsmuster legen nahe: Was Demokratie ist, lässt sich hier nicht oder allenfalls nur in der Abstraktheit flackernder Nebelkerzen fassen. Als globales Fahnenwort ist Demokratie auf allen Ebenen fest etabliert - nicht erst seit dem Ende des Kalten Krieges. Demokratie meint im politisch-medienöffentlichen Diskurs meist implizit (nur manchmal explizit) und pauschal the western way of life, der als Kontrastfolie zur Einordnung von "Antidemokraten" (also all jenen, die den eigenen Interessen zuwiderhandeln) fungiert. Darum lässt sich über Demokratie als solche heute auch nicht mehr streiten: wer Systemfragen stellt, exkludiert sich aus dem Kreis der "Freiheitlichdemokratischen Grundordnung", ist Extremist oder Meckerer (früher konnte man ihm sagen: dann geh doch rüber! Heute droht ihm Demokratisierung, das heißt im Nato-Jargon: "Befriedung" mit aller militärisch-okkupierender Gewalt). 
"Demokratie" - zwischen Kampfbegriff und Nebelkerze

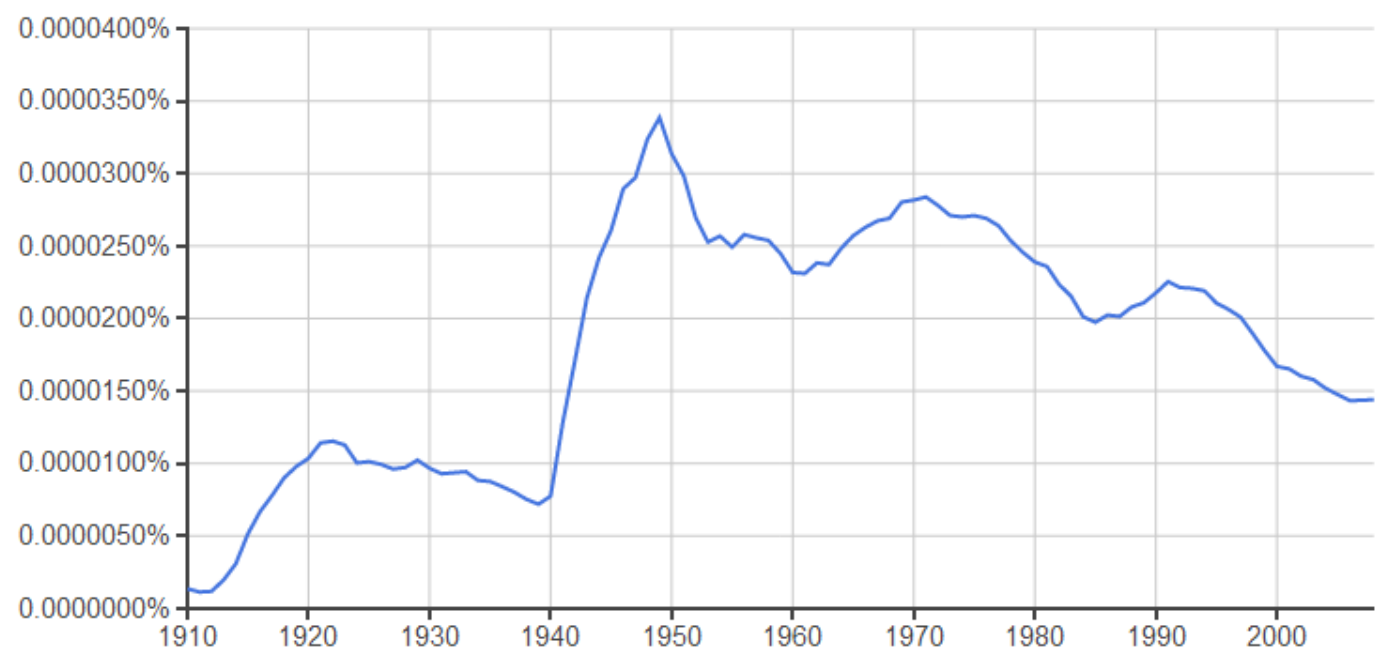

Abbildung: Relative Frequenzen der Zeichenketten (case insensitive) Demokratie bedeutet/heißt in googlengrams (ngrams des google-Books-Archiv); Kurve bleibt weitestgehend erhalten, wenn man Quasisynonyme wie Partizipation (in den 2000ern leicht zunehmend) oder Mitbestimmung berücksichtigt

\section{Linguistische Beiträge zur Demokratieforschung: eine programmatische Skizze}

In den Sprach-, Medien- und Kommunikationswissenschaften spielt das Thema Sprache und Demokratie (ebenso wie das Thema Menschenrechte, vgl. Vogel 2014a) im engeren Sinne bislang nur eine untergeordnete und vor allem rein deskriptive Rolle. Thema dieser Arbeiten ist etwa die Begriffsgeschichte von Konzept und/oder Ausdruck Demokratie (vgl. Kämper 2009; Kämper/Haslinger/Raithel 2014; Kilian 2003, 1997), Kommunikationsformen im Parlament (vgl. Dörner/Vogt 1995; Burkhardt 2000), politischer Wortschatz (vgl. Klein 2002, 2005; Busch 2005) u. ä. Wie aber könnte ein sprachwissenschaftlicher Beitrag zu einer normativen Demokratieforschung aussehen und welche Fragen wären zu bearbeiten?

(1) Voraussetzung für eine aussagekräftige linguistische Demokratieforschung wäre zunächst die Entwicklung eines eigenen Demokratie-Verständnisses, das Demokratie nicht lediglich als Staats- oder gar Lebensform in ihrer verfahrenstheoretischen Idealisierung als gegeben unterstellt. Dies scheint aber der Großteil bestehender Beiträge zu tun, wenn sie - zudem mit bestimmtem Artikel - etwa von "der demokratischen Sprache" (so im Titel bei Kilian 1997) in der Bundesrepublik schreiben. Zugespitzt formuliert: "Demokratisch" ist die Sprache dort nur deshalb, weil sie in die Zeit der "parlamentarischen Demokratie" (oder ihrer Vorbereitung) fällt, also zur sinngebenden "Epochensprache" stilisiert wird. Nur vereinzelt werden Fragen nach möglichen Voraussetzungen für eine "lebende Demokratie" diskutiert. Wie passt aber zu einer "demokratischen Sprache", dass eine Kanzlerin folgenlos Freude über die (wie sich herausstellt: inszenierte; vgl. www.heise.de/tp/artikel/44/44947/1.html [27.05.2015]) Ermordung bzw. öffentlichkeitswirksame Hinrichtung eines Menschen (hier: Osama bin Ladens) öffentlich äußern kann (vgl. www.spiegel.de/politik/deutschland/freude-ueber-bin-ladens-todmerkel-mueht-sich-um-schadensbegrenzung-a-760662.html [27.05.2015])? Welche Bedeutung hat eine "demokratische Sprache" jenseits der bildungsbürgerlichen Mittelschicht, bei sozialen Eliten (etwa Oligarchen, die - anstatt sie wie bisher nur von außen zu kaufen - sich mittlerweile einfach selbst in die Regierung wählen lassen) oder aber dem Drittel oder (wie bei der Bürgerschaftswahl 2015 in Bremen oder bei den EU-Wahlen 2004) der Hälfte der Gesellschaft, das sich Wahlen verweigert? Und wie können unter dem Losungswort "Sicher- 
heit" heute Milliarden Menschen voll überwacht werden (NSA-Skandal) und als Antwort darauf neue Überwachungsmaßnahmen (Vorratsdatenspeicherung 2015) eingeführt werden?9

Unter "Demokratie" möchten wir dagegen in Anlehnung an Fields und Narr (1992) sowie in einem weiten und forschungspraktischen Sinne eine soziale Interaktionsform verstehen, die dazu dient und historisch situativ dazu geeignet ist, individuelle und potentiell divergierende Bedürfnisse - allen voran das Bedürfnis nach Handlungsfreiheit, sozialer Anerkennung, kontextsensitiver Gleichheit (insb. mit Blick auf Güterverteilung) und persönlicher Integrität -, in sozialen Gruppen gewaltfrei und heterarchisch zu vermitteln. Eine solche, als "demokratisch" bezeichnete Interaktionsform erfordert, dass die Beteiligten habituell sowie verfahrensmäßig in die Lage versetzt werden, sich auf Basis von überprüfbaren Informationen eine kollektivierbare Meinung zu bilden und ihre damit verbundenen Bedürfnisse adressatengerecht kommunizieren zu können (vgl. Vogel 2014a). Dieses Demokratieverständnis soll nicht als idealisierte Chimäre über die erfahrbare Praxis gelegt werden (wie es zahlreiche politik- oder rechtswissenschaftliche Einführungen in das politische System Deutschlands allzu oft tun). Es soll vielmehr als orientierendes konzeptionelles Such- und Orientierungsraster für ein empirisches sprach-, medien- und kommunikationswissenschaftliches Forschungsprogramm dienen. Hierbei wären folgende Fragenkomplexe zu bearbeiten bzw. - soweit hierzu bereits unter anderer Fahne wehende Arbeiten vorliegen neu zu sortieren:

(2) Wie erfolgt individuelle sowie kollektive Meinungsbildung unter den gegenwärtigen gesellschaftlichen Bedingungen, unter dem Einfluss 1) verschiedener Partialinteressen und 2) sich verändernder medialer Resonanzräume in der Massendemokratie? Partialinteressen von privaten (individualisierten) Akteuren, wirtschaftlichen Akteuren (unter den Maximen der Profitmaximierung), staatlich-behördlichen Akteuren (von Öffentlichkeitsabteilungen der Bundesregierung bis zu Geheimdiensten), parteipolitischen Interessensgruppen, juristischen Akteuren, außerstaatlichen, zivilgesellschaftlichen Akteuren (vom Verein bis zum ThinkTank) und Medienagenten (Journalisten, PR-Agenturen u. ä.) stehen mal im Konflikt zu einander, mal sind sie voneinander abhängig ("Politainment" und "Mediokratie") und/oder gehen zeitweise funktionale Allianzen ein. ${ }^{10}$ Die tatsächlichen und potentiell alternativen Verfahren oder Praxen der Meinungsbildung differieren ferner je nach medialer Konstellation, von den klassischen Einwege-Medien wie Printzeitung und Hörfunk über veröffentlichte Parlamentssitzungen bis hin zu virtuellen Räumen auf Basis von Social Software. So findet kommunikativer Austausch über Youtube unter anderen Vorzeichen statt als etwa über Wikis. Welches Potenzial der Informationskollektivierung bzw. Handlungsorientierung ("Arabischer Frühling") und -manipulation (von verdeckten Werbekampagnen und "Imagemaking" bis Cyber-Armeen à la "77 th $^{\text {Brigade"11 }}$ Großbritanniens oder IS-Hackern (vgl.

\footnotetext{
9 Man könnte noch vieles anschließen, nur eines sei hier betont: "Spitzengespräche" mögen vielleicht ein "effizientes Instrument bei der Lösung von Konflikten im Rahmen informellen Regierungshandelns" sein, sie sind aber natürlich nicht im geringsten "demokratisch" (so aber Pape 2005: 125).

10 Für letzteres, die Kooperation von Polizeibehörden, Politakteuren und Medien gibt die Studie von Dießelmann in diesem Heft ein anschauliches Beispiel. Ein anderes Beispiel lässt sich derzeit (Mai 2015) in der Presse verfolgen, nämlich die Zusammenarbeit von Geheimdiensten (hier NSA und BND) und global agierenden Wirtschaftsakteuren (Wirtschaftsspionage).

${ }^{11}$ Vgl. theguardian vom 31.01.2015 (http://gu.com/p/45cfq/sbl [06.05.2015]).
} 
"Demokratie" - zwischen Kampfbegriff und Nebelkerze

www.spiegel.de/netzwelt/web/islamischer-staat-is-hacker-legen-fernsehsender-tv5mondelahm-a-1027631.html [06.05.2015]) bieten Online-Foren ${ }^{12}$ ? Oder wie ließen sich Ansätze der liquid democracy für eine E-Demokratie weiterentwickeln und bekannte Probleme lösen (vgl. darkbln 2011; Adler 2013)? Gerade im Kontext transnationaler Konflikte und kriegerischgewaltvoller Auseinandersetzungen - wie mit dem Ukraine-Konflikt derzeit vor der eigenen europäischen Haustüre (vgl. Vogel/Luth/Ptashnyk i. E.) - ist es notwendiger denn je, durch sprach-, kommunikations- und medienwissenschaftliche Analytik zu sortieren und im Falle von Propaganda (gleich welcher Couleur) aktiv im Diskurs zu intervenieren. Zu diesem Zweck bedarf es auch neuer Methoden der Analyse und Dokumentation von staatlicher Gewalt. Wie eine solche zivilgesellschaftlich (d. h. außerbehördlich) zu forcierende Medienforensik aussehen könnte, illustriert etwa der Ansatz einer "Architecture Forensic" von Eyal Weizman (London), der unterschiedlichste mediale Daten und neue technische Verfahren der Modellierung zur Aufklärung von umstrittenen Gewaltereignissen zusammenführt (vgl. www.forensic-architecture.org [07.05.2015]).

In diesem Sinne wäre auch weiter zu fragen nach neuen Strategien, wie im medialen Wust überwiegend ungeprüfter und/oder unüberprüfbarer Informationsweitergabe dennoch qualitativ hochwertige, argumentierende (statt persuasiv-polemisierende) Informationskanäle gefunden, gestützt und intelligent genutzt oder didaktisch vermittelt werden können. Spiegel-Online zählt nicht nur bei jüngeren Generationen zu den Online-Medien mit höchsten täglichen Besucherzahlen, die Informationsqualität des Portals baut aber im Wesentlichen auf ungeprüfter Übernahme (Copy \& Paste) von Agentur- und Boulevardmedien auf - das aber immer schnell und aktuell. Angebote, die den medialen Wust auf einer Metaebene kategorisieren und filtern, wie etwa die von Albrecht Müller und Wolfgang Lieb herausgegebenen "Nachdenkseiten" (www.nachdenkseiten.de), sind kaum bekannt. In diesem Sinne wird es immer wichtiger, nicht nur Heranwachsenden (über die Schüler wird immer gescholten; man blicke aber Studierenden und Studierten nur über die Schulter) möglichst früh eine Medienkompetenz zu vermitteln, die über Twitter und Facebook hinausgeht und eine Sensibilität für das "Web of trust"13 aufbaut.

(3) Wo zentrale Entscheidungsfindungsprozesse zur Regelung des gesellschaftlichen Zusammenlebens von wenigen, milliardenschweren "global Playern" dominiert werden, bedarf es heute einer zuverlässigen Ökonomie der Demokratie-Inszenierungen. Gemeint sind diskursive Routinen und Praxen, die zwar offiziell zur Legitimierung von Entscheidungsträgern dienen sollen, die aber faktisch nicht dazu geeignet sind, gesellschaftliche Teilhabe (und das heißt immer auch: aufwendige Meinungsbildungs- und Aushandlungsprozesse) sozialer Gruppen zu gewährleisten. An die Stelle von Aushandlung tritt dabei immer häufiger eine Zwangsinklusion per Konsensfiktion, von einwandsimmunen Fahnenwörtern (Inklusion, Autonomie - wer ist schon für "Ausgrenzung" oder "Freiheitsbeschränkung"?) über inszeniertes Expertentum (Wer positioniert sich schon gegen Wirtschaftsweise und wissenschaftlichem

\footnotetext{
12 Zum Politikchat vgl. Diekmannshenke 2005.

13 Das Konzept "Web of trust" stammt eigentlich aus der Kryptographie und zielt auf die Idee, die Authentizität von Nachrichten durch ein passives soziales Netzwerk abzusichern. Dieser Gedanke ließe sich auch in neue Medieninformationskonzepte übertragen, etwa zur Organisation von hochwertigen Informationsknoten im medialen Hintergrundrauschen.
} 
Autoritätstopos) bis zu alltäglicher alternativlos-Rhetorik. Wo keine wirklich konfligierenden Meinungsunterschiede herrschen (weil die Sprecher für die wichtigen Entscheidungsprozesse verlustlos austauschbar sind), aber doch eine "Wahl" vorgegaukelt werden muss, werden im "Wahlkampf" Fernsehduelle und Elefantenrunden zur Schau gestellt. Nur selten bricht die Inszenierungsbühne zusammen, etwa wenn sich CSU-Wähler als verpflichtete Erntehelfer entpuppen ${ }^{14}$ oder ein auf die Diskursperipherie (Zuschauerbank) verwiesener Flüchtlingsretter den wohlstudierten Konsensbrei einer Günter-Jauch-Studio"diskussion" sprengt ${ }^{15}$ (vgl. zum Wandel meinungsbildender TV-Diskussionen schon Holly 1993; Holly/Schwitalla 1995). Zur Inszenierung von Teilhabe gehören dann auch die verschiedenen Muster der diskursiven Einhegung von solchen Ausnahmefällen. So gibt die kolportierte TV-Diskussion kaum zwei Tage Anlass zur allgemeinen, folgenlosen Empörung. Abgesehen davon, dass Flüchtlingsräte und Amnesty International seit Jahren medial ungehört auf die Problematik ertrinkender Mittelmeerflüchtlinge aufmerksam machen, wird das "Medienevent" (Sternstunde der Medien) personalisiert und alsbald vergessen. Die Politik nimmt den Resonanzraum auf und codiert das Thema kurzerhand und einwandsimmun um in die Losung "Mehr Schutz für die Flüchtlinge vor Schleuserbanden" - und legitimiert damit einen weiteren Ausbau von Frontex. ${ }^{16}$ Systematisch zu untersuchen wären auch die diskursiven Immunsysteme nicht nur totalitärer Staaten, sondern auch sich davon abgrenzender parlamentarischer "Demokratien". Zahlreiche Beispiele hier bietet etwa der deutsch-europäische Umgang mit Volksbegehren im Kontext der Wirtschaftskrise. Man erinnere sich etwa an die allgemeine Empörung über die Erklärung des damaligen Ministerpräsidents Papandreou 2011, er wolle die griechische Bevölkerung über die Troika-Maßnahmen abstimmen lassen. Auch die Einhegungspraxen im Kontext des irischen Referendums zum Fiskalpakt 2012 gäben interessante Einblicke in das Zusammenspiel transnational agierender hegemonialer Akteure.

Ein letztes, gerade aus linguistischer Perspektive interessantes Beispiel für Demokratieinszenierungen ist das Thema "Leichte und einfache Sprache" (vgl. dazu auch der Beitrag von Bettina Bock). Weil der partizipatorische Prozess über Sprache konstituiert und aufrechterhalten wird, setzt politische Teilhabe sprachlich-kommunikative Teilhabe voraus. Und weil jeder weiß, dass schon ein Strafmandat oder eine Steuerbescheinigung völlig unverständlich einher kommen, muss Partizipationsfähigkeit in der Politik durch "einfache" oder "leichte Sprache" gesichert und gefördert werden. So der Grundgedanke, der sich zwanglos in den Großdiskurs über "Inklusion" einordnet. Die Denkfigur ist einfach und praktisch einwandsimmun. Wer kann schon etwas gegen die Erleichterung sprachlicher Teilhabe am politischen Geschehen sagen? Aber schon auf den zweiten Blick wird die Absurdität dieses Vorhabens unübersehbar. Leiden Wahlkämpfe etwa an der bildungssprachlichen Komplexität der Plakate, Internetauftritte oder Fernsehdebatten? Gewiss nicht, die Primitivisierung der öffentlichen Rede ist ein Fakt. Wenn es wirklich Sprachbarrieren sind, welche die Masse der Bevölkerung von politi-

\footnotetext{
14 Süddeutsche vom 19.09.2015 (www.sueddeutsche.de/bayern/ermittlungen-wegen-wahlmanipulation-der-duftdes-spargels-1.1928123 [07.05.2015]).

15 Vgl. Zeit-Online vom 20.04.2015 (www.zeit.de/news/2015-04/20/medien-schweigeminute-bei-jauch-fuerertrunkene-fluechtlinge-20102012 [07.05.2015]).

${ }^{16}$ Die "Europäische Agentur für die operative Zusammenarbeit an den Außengrenzen der Mitgliedstaaten der Europäischen Union" (Frontex), deren teilweise militärische Praxis von Menschenrechtsorganisationen als Verstoß gegen internationales Recht bewertet wird (vgl. etwa das Gutachten des ECCHR 2007; www.amnesty.de/umleitung/2007/deu01/047 [07.05.2015]).
} 
"Demokratie" - zwischen Kampfbegriff und Nebelkerze

scher Teilhabe fernhalten, wieso konstatieren dann die Sozialwissenschaftler seit zwei Jahrzehnten, dass ausgerechnet die Bildungsschichten und Intellektuellen im hegemonialen Apparat der öffentlichen Meinung kaum mehr präsentiert sind? Während man einem Rundfunkvortrag von Adorno in den 60er Jahren andächtig gelauscht hat, wären solchermaßen "intellektuelle" Texte in der heutigen Medienlandschaft per se Satire. Und ausgerechnet in dieser Konstellation will man die zuvor als bildungsfern verhöhnten Schichten durch "Leichte Sprache" zur Teilhabe an der Macht befähigen? Wer den Schaden hat, braucht bekanntlich für den Spott nicht zu sorgen. Die Sprache der Macht ist so leicht und einfach, dass sie wirklich jeder versteht. Stefanowitsch (2014) gibt mit Recht zu bedenken, dass beherrschte Registervielfalt und Sprachausbau die Denkfähigkeit des Einzelnen tangieren. Wäre es nicht womöglich lohnender, sich für eine umfassende Beherrschung ausgebauter sprachlicher Register stark zu machen, anstatt einer weiteren Primitivisierung des öffentlichen Sprechens das Wort zu reden?

Die Leichte Sprache ist zum einen Symbolpolitik. Die Eliten legen ein Programm auf, das ihre Sorge um die Partizipationsmöglichkeiten der breiten Massen werbewirksam zur Schau stellt. Ändern tut sich dadurch nichts, sieht man davon ab, dass ein politischer Propagandist der Leichten Sprache zu einer wunderbaren fiktiven moralischen Gemeinschaft gehört und reiche Imagegewinne für sich verbuchen kann. Zum anderen wird stillschweigend so getan, als sei die Leichte Sprache nur eine technische Übersetzung ihres "komplizierten" Pendants. Aber "leichter" ist, wie die Perspektivität von Sprache lehrt, eben nicht nur "nicht schwer", sondern immer auch anders. Nicht nur die Sprache, auch die Erklärungs- und Begründungszusammenhänge werden dann plötzlich überraschend "leicht". Die Devise: Mitreden, nicht mitdenken, erst recht nicht mitentscheiden.

(4) Mit dem letztgenannten wird auch ein eigenständig zu entwickelnder Bereich linguistischer Demokratieforschung gestreift, nämlich die Frage, welche habituellen und gesellschaftlichen Voraussetzungen gegeben sein müssen, um Individuen zu "demokratischem Sprechen" und damit zur praktischen Teilhabe an gesellschaftlichen Entscheidungsfindungsprozessen im oben (1) skizzierten Sinne zu befähigen (vgl. dazu Neuland 2005; Vogel 2014a). Damit angesprochen ist der gesamte Komplex der sprachlich-kommunikativen sowie auch inhaltlich grundständigen Aus- und Weiterbildung von der Wiege bis zur Bahre. Die Fähigkeit, die eigenen Bedürfnisse situativ angemessen zu vermitteln, ist bekanntlich eine komplexe Angelegenheit: Sie erfordert nicht nur das Bewusstmachen eigener und fremder Interessen, sondern auch Registersensibilität, also Empathie und Antizipationsfähigkeit im Hinblick auf potentiell verletzendes oder gar gewaltsames Sprechen und vieles andere. ${ }^{17}$ So verstandene Kommunikationsfähigkeit ist mehr als auf Arbeitsmarktbedürfnisse abgerichteter "skill". Sie ist menschenrechtliches Grundbedürfnis. Es wäre Aufgabe von Sprach-, Medien- und Kommunikationswissenschaftlern, sie nicht nur didaktisch zu begleiten, sondern auch gegenüber exkludierender Bildungspolitik (mehrgliedriges Schulsystem, Schulabgang nach 9 Jahren, Eliten- und Exzellenzprogramme vom Kindergarten an usw.) zu vertreten. Die Schreibfor-

\footnotetext{
17 Einiges lernen ließe sich hierbei nicht nur allgemein von der empirischen gesprächs- und interaktionsanalytischen Konfliktforschung (im Überblick hierzu: Schwitalla 2001), sondern vor allem auch aus detailreichen Fallstudien zur erfolgreichen Konfliktbewältigung in kleineren, politisch agierenden Gruppen (vgl. etwa Läzer 2000 und schon früher Schwitalla und Keim 1989).
} 
schung leistet hierzu bereits wertvolle Dienste (vgl. etwa das Projekt zur eristischen Literalität an der Universität Gießen).

Die Folgen mangelnder Kommunikationsfähigkeit lassen sich eindrücklich im Kontext der Fach-Laien-Kommunikation vor Gericht oder im Kontext von Asylbewerbungsverfahren studieren. Vor allem Flüchtlinge sind hierzulande häufig - im wörtlichen Sinne - sprachlos. Sie sind nicht nur den schon für Deutschkundige kaum durchschaubaren Verfahren hilflos ausgeliefert; sie erhalten auch keine doch grundrechtlich "garantierte" kommunikative Unterstützung in medizinischen Notfällen. So werden schwerste psychische und physische Erkrankungen, teilweise Folge von Verfolgung und Flucht, nicht oder zu spät erkannt, werden falsche Mittel oder bis zur Abschiebung lediglich Beruhigungsmittel und Aufheller verschrieben.

Aber auch unter jenen, die seit ihrer Geburt einen deutschen Pass tragen, ist die Rate der Analphabeten nach wie vor hoch. Die Leo-Studie der Universität Hamburg (vgl. Grotlüschen/Riekmann 2011) sprach 2011 von 7,5 Mio. (also mehr als 14 \%) funktionalen Analphabeten unter Erwerbsfähigen in Deutschland, d. h. Personen, die Mangels Beherrschung der Schriftsprache teilweise erhebliche Probleme bei der Bewältigung ihres Alltags haben. Weitere 13 Mio. Menschen (25\%) zeigten mangelhafte Schreibfähigkeit.

Was den einen fehlt oder verwehrt wird, wird anderen praktisch systematisch abtrainiert. Am Ende der sprachlich-kommunikativen Enteignung steht der durchrationalisierte Sprachroboter (vgl. Boutet 2012) in indisch-ausgelagerten Call-Centern, der nicht Denken, sondern nach festgelegten Routinen kommunikativ übertölpeln können muss.

(5) Schließlich ist nach der Rolle, ja der Mitverantwortung der Sprach-, Medien- und Kommunikationswissenschaften für den Zustand der öffentlichen, medialen und politischen Debatten zu fragen. Auch die Linguistik wird sich dem Sog der Medialisierung, Kommerzialisierung und Politisierung wissenschaftlicher Themen (vgl. Weingart 2001) auf die Dauer nicht entziehen können. Materielle und symbolische Ressourcen des Faches hängen davon ab, dass die Linguistik einen wahrnehmbaren (und möglichst unbequemen!) Beitrag zur Reflexion der sprachlichen Verhältnisse leistet. Das "Unwort des Jahres" wird auf Dauer nicht hinreichen. Von der "Bringschuld" der Geisteswissenschaften (und natürlich auch der Linguistik) gegenüber der Gesellschaft ist neuerdings oft die Rede. Das suggeriert, dass es mit der traditionellen Wahrheitssuche und mit der viel beschworenen Autonomie der Wissenschaften womöglich nicht mehr sehr weit her ist. Aber wer ist eigentlich "die Gesellschaft", der die Linguistik etwas schuldet, wenn nicht die Ansammlung von kompakten Akteuren, die sich erfolgreich als hegemonial etabliert haben? Was will die Wirtschaft als alt-neue Hegemonialmacht von den Linguisten? Was wollen wir selbst, jenseits der (selbstverständlichen) Dienste, die Sprachwissenschaftler z. B. den Juristen bei der verständlichen Formulierung von Gesetzen und Verordnungen leisten? Wie kann sich ein Fach, das (nach einer kurzen Phase der heillosen Überschätzung und Erwartungsüberfrachtung in den 60er und 70er Jahren des vorigen Jahrhunderts) längst wieder in dem Ruf steht, Glasperlenspiele zu pflegen, in die Verhältnisse aktiv einmischen, von deren sprachlich-kommunikativer Ökonomie es selbst abhängt? Es muss sich nachgerade einmischen! - Verdienste um die Demokratie könnte sich die Sprachwissenschaft daher erwerben, wenn sie sich dazu entschließen würde anzuerkennen, dass sie selbst ein fachkundiger Akteur mit Expertenstatus auf dem öffentlichen Kampffeld politischmedialer Kommunikation ist: "Linguistik als Kampfsport" (Vogel 2014a). 


\section{$4 \quad$ Die Beiträge dieser Ausgabe}

Natürliche Sprachen - so der US-amerikanische Sprachphilosoph und Literaturkritiker Kenneth Burke - versorgen ihre Sprecher mit dramaturgischen Ressourcen, die es erlauben, winzige Veränderungen revolutionär und veritable Umwälzungen normal und alltäglich aussehen $\mathrm{zu}$ lassen. "It is the normally prayerful use of language, to sharpen up the pointless and blunt the too sharply pointed", schreibt Burke (1969: 393) mit Blick auf die radikal antikapitalistische Regierungsrhetorik der frühen Roosevelt-Jahre. In der Tat lässt sich ein solches Wechselspiel zwischen der rhetorischen Normalisierung radikaler Einschnitte in demokratische und soziale Rechte und der komplementären Überdramatisierung alltäglicher demokratischer Praktiken auch heute allenthalben beobachten: Wenn Lokführer, Kindergärtner oder Piloten streiken, dann droht dramatische Denormalisierung und der Ruf nach Begrenzung des Streikrechts auf ein der Volksgemeinschaft bekömmliches Maß wird lauter. Umgekehrt fehlt es nicht an der "Dedramatisierung" staatlicher und wirtschaftlicher Rechtsbrüche - mit Verweis auf den außerkonstitutionellen Höchstwert "Sicherheit" oder auf die Wirtschaftsinteressen eines "Exportweltmeisters". Das sprachliche Verhältnis, um das es in solchen Dingen geht, bezeichnet Burke als scene-act-ratio, als eine Art dialektischer Korrespondenz zwischen der (sprachlich gefassten) "Szene" und den (mehr oder weniger angemessenen) Handlungen, die in dieser Szene ausgeführt werden. Klar: Eine als Krise, Katastrophe, Ausnahmezustand diagnostizierte Szene erfordert andere Handlungen als eine ganz "normale" Szene. In einer Situation umfassender "terroristischer Bedrohung" sind ganz andere Handlungen geboten und legitim als unter den Bedingungen von business-as-usual. Dieses Verhältnis kann deterministisch veranschlagt werden: Weil die Situation dramatisch war, mussten auch dramatische Maßnahmen ergriffen werden. Sie kann aber auch hortatorisch veranschlagt werden: Weil die Situation dramatisch ist, sollten auch dramatische Handlungen und Kompetenzausweitungen erwogen werden. Einmal erscheinen die Akteure als Gefangene der dramatischen Situation, das andere Mal erscheinen sie im Gegenteil befreit von den "normalen" Zwängen und Begrenzungen des Handelns. In dieser Konstellation liegt der unwiderstehliche Charme, den das Regieren im Krisenmodus für die Mächtigen bereithält. Sie können ihre Handlungsspielräume erweitern und diese hinzugewonnen Freiheiten zugleich als notwendige Folge einer geteilten krisenhaften Situationsdefinition verkaufen.

Aufschlussreich für die Diagnose des Verhältnisses von Sprache und Demokratie sind freilich auch andere ratios (im Sinne von Burke 1969), nicht zuletzt das Verhältnis von agent und scene, um das es im Beitrag von Ruth Wodak geht. Wo Migranten oder Flüchtlingen "Integrationsunwilligkeit" bescheinigt wird, da geht es um eine Motivzuschreibung für ein Fremdkollektiv, das für die adressierten Eigenwerte als fremd und bedrohlich kodiert wird. Die Abwehr dieses Fremdkollektivs durch die Eigengruppe erscheint dann umgedreht als Abwehr der eigenen Werte durch das Fremdkollektiv. Ruth Wodak verfolgt den Weg dieser Motivzuschreibung in der österreichischen Politik von den FPÖ-Anfängen in den 90er Jahren bis hin zur Übernahme des Topos durch die Mitteparteien in jüngster Zeit. Im Dreieck von Neoliberalismus, Populismus und kritischer Öffentlichkeit wird die "Integrationsunwilligkeit" dadurch konnotativ mittefähig, dass sie indexikalisch mit Ereignissen in Verbindung gebracht wird, deren negative Bewertung auch außerhalb rechtspopulistischer Milieus unterstellt werden kann: Es sind dies einerseits die bekannten Schulprobleme (gemischter Sport- und Schwimmunterricht, Ablehnung weiblicher Autoritäten usw.), andererseits die wirtschaftli-

ISSN 1615-3014 
chen Topoi der neoliberalen Ideologie, die persönliche "Freiheiten" an ökonomische Effizienz und ökonomisches Funktionieren koppeln und Migration auch vor diesem Hintergrund als fremd und gefährlich kodieren. Beide Motivkreise erweisen sich als höchst geeignet, dem Vorwurf der "Integrationsunwilligkeit" den Weg in die Mitte zu bahnen. Es sind, beiläufig, exakt die Motivkreise, auf die auch Sarrazin in Deutschland mit einigem Erfolg gesetzt hat.

Um die grammatischen und rhetorischen Ressourcen des Demokratiebegriffs geht es im Beitrag von David Salomon. Politisch wird ein Begriff dadurch, dass er öffentlich Zustimmung, Ablehnung, Polarisierung kommandiert und so zum Aufbau oder zur Verteidigung von Machtpositionen, Bündnissen, Feindverhältnissen usw. eingesetzt werden kann. So setzt die verbreitete Diagnose "postdemokratischer" Verhältnisse unbedingt auf den fortdauernd guten Klang des Fahnenwortes "Demokratie", Post-X geht eigentlich nur, wenn X noch über beträchtliches Zustimmungspotential verfügt. Salomon zeigt in seinem Beitrag, dass es nach dem rasanten Siegeszug der (bis zum Beginn des vorigen Jahrhunderts als Pöbelherrschaft durchweg negativ konnotierten) Demokratie in der Hauptsache um die Trennung bzw. Amalgamierung politischer Beteiligung und sozialer Gerechtigkeit geht. Wer bis in die neoliberale Gegenwart positiv von "Demokratie" spricht, der meint fast immer die Einheit von (mehr oder minder) freien Wahlen und entfesselten Kapital- und Marktregimes. Und was geschieht, wenn diese beiden "Mächte" in Gegensatz zueinander geraten, das kann man derzeit in Griechenland beobachten, wo EU, IWF und Bundesregierung unisono von der griechischen Regierung fordern, als allererstes habe sie dem eigenen Wahlvolk zu vermitteln, dass Wahlversprechen keinesfalls gehalten werden können! Salomons Plädoyer für einen programmatisch sozialen Demokratiebegriff steht in der Tradition Wolfgang Abendroths - und im radikalen Gegensatz zu allen Versuchen der Entkopplung von Demokratie und Gesellschaft. Letztere sind charakteristisch für die neoliberale Ideologie, und die radikalste Version dürfte Margaret Thatcher vorgetragen haben mit dem oft zitierten Satz, dass es so etwas wie eine "Gesellschaft" gar nicht gebe, sondern nur Individuen und Familien. Wiederum in der Terminologie von Kenneth Burke geht es also in David Salomons Beitrag um die Frage, ob die sozialen Belange integral zu einer demokratischen Szene gehören oder ob sie in einer solchen kontingente Rand- oder Störgrößen bilden. Als rhetorischer Strategiekern verkörpert der Ausdruck "Demokratie" bewegliche Handlungsoptionen politisch-sozialer Akteure in einem umkämpften Feld.

Um rhetorische Strategien rechter Meinungsführerschaft geht es im Beitrag von Maria Stopfner. Theoretisch und methodisch ist der Beitrag basiert in der klassischen Forschung zu den sozial-kommunikativen Voraussetzungen von "Meinungsführerschaft". Deren Erkenntnisse (wer Einfluss nehmen will, muss sich selbst in der "Umgebung" der Adressaten verorten usw.) überträgt die Autorin produktiv auf die Verhältnisse von Online-Diskussionen. Wie dort rechte "Kompetenz" und "Identität" inszeniert werden, zeigt die Autorin mit Hilfe eines Korpus, bestehend aus 1047 Kommentaren aus dem Jahr 2009, die in den Foren der österreichischen Zeitungen Die Presse und Krone gepostet worden sind. Zurückgreifend auf Arbeiten von Wodak, Reisigl und Wenger (communities of practice) rekonstruiert die Autorin, wie sich rechte Randgruppen erfolgreich als realistische "In-Group" in Szene setzen. Die organisierte Rechte, so die Autorin, konstituiert und inszeniert sich in Netzdebatten als kompetenter und besonnener Akteur in einer verdummten und ideologischen Szene. 
"Demokratie" - zwischen Kampfbegriff und Nebelkerze

In der philologischen Tradition eines Viktor Klemperer verortet sich der Beitrag von Martin Haase: Auch das, was die Sprecher in ihren Äußerungen zu verbergen suchen, kommt unweigerlich in ihren Texten zum Ausdruck. Im Kern gehören die von Haase aufgezeigten Praktiken in den Bereich des taktischen oder strategischen "Framing". Aufschlussreich ist das Einstiegsbeispiel einer Formulierung aus einem Koalitionsvertrag, bei der sich beide Parteien verpflichten, eine Sache höchst entschlossen anzugehen, bei der aber bereits an den Formulierungen zu erkennen ist, dass sie möglichst still beerdigt werden soll. Der Beitrag von Haase gleicht einer lehrreichen und unterhaltsamen Rundwanderung durch einige Felder eingespielter strategischer Ausdrucksselektion. Einmal geht es darum, nichts zu sagen, was später vielleicht juristisch als Lüge gewürdigt werden könnte (Guttenberg), dann wiederum kommuniziert bereits die veränderte Nennung (vom "Nacktscanner" zum "Körperscanner der zweiten Generation"), wie eine Neuerung dem Publikum durch strategische Umbenennung schmackhaft gemacht werden soll.

Einer kollektivsymbolischen Analyse sprachlicher und optischer Bilder ist der Text von Dorothee Meer verpflichtet. Die Autorin liefert eine akribische Analyse der Berichterstattung und Kommentierung zum polizeilichen Abbruch eines Hungerstreiks von Flüchtlingen am Münchener Rindermarkt in den beiden Zeitungen $S Z$ und $F A Z$. Der Schwerpunkt liegt auf dem Verhältnis von Abbildungen einerseits und Sprachbildern andererseits. Der Fall ist insofern exemplarisch und aufschlussreich, als es zu einem medienöffentlichen Wettstreit von Moralisierung und Gegenmoralisierung gekommen ist, bei dem am Ende das Symbol der "Schwangeren" ausschlaggebend war. Die "fürsorgliche" Polizei beendet das verantwortungslose und selbst- wie kindswohlgefährdende Verhalten der Schwangeren und wird als moralisch überlegen wahrgenommen. Bilder von Schwangeren taugen ansonsten eher dazu, Sympathie mit dem Anliegen der Abgebildeten zu wecken und gewaltsame polizeiliche Lösungen zu entmutigen: Polizeiknüppel gegen einen schwangeren Bauch sind moralisch immer im Unrecht (und machen sich auf Medienbildern denkbar schlecht!). Und muss die Not nicht furchtbar groß sein, wenn sich eine Schwangere an einem Hungerstreit beteiligt? Wie diese moralische Konstellation umgedreht wird, das legt der Artikel schlüssig frei. Die Polizei "rettet" am Ende in der medialen Darstellung die Flüchtlinge mit ihrem moralisch berechtigten Anliegen vor der Instrumentalisierung durch skrupellose "Linksradikale", deren mediale Insbildsetzung methodisch untersucht wird. Der Text enthält zahlreiche weiterführende Thesen und Beobachtungen zum Text-Bild-Verhältnis in der politischen Berichterstattung und ist insofern von hohem Wert für alle, die im Feld der linguistischen Diskursanalyse arbeiten.

Eine steile Diskurskarriere hat in den vergangenen Jahren die hoch moralisierte Figur der "Inklusion" hingelegt (vgl. Knobloch 2015). Der demonstrative Versuch, auch Schwache und Benachteiligte in Beruf, Bildung und Politik mitzunehmen und einzubeziehen, adelt jeden, der ihn publikumswirksam unternimmt. Ob er den so "Eingeschlossenen" ebenfalls nützt, steht freilich auf einem anderen Blatt. In der demokratisch-politischen Szene marschiert die Inklusion hinter dem Fahnenwort "Leichte Sprache". Der Beitrag von Bettina Bock trägt zusätzlich den Anspruch der "barrierefreien Kommunikation" im Titel, der nicht ganz frei von unheimlichen Konnotationen zu sein scheint. Am Beispiel zweier auf Verständlichkeit überarbeiteten Versionen des SPD-Parteiprogramms prüft die Autorin kritisch den demokratischen Inklusionsanspruch der Leichten Sprache. Der könnte sich nämlich leicht als nachhaltige Exklusion entpuppen, stigmatisiert Leichte Sprache doch diejenigen, welche (vermeintlich) auf 
sie angewiesen sind, womöglich nachhaltiger als Unverständnis für das reguläre SPDProgramm! Und umgekehrt verleiht Leichte Sprache denen, die sich politisch und konzeptionell um sie bemühen, das moralische Image, das jedweder Einsatz für die Partizipation von Benachteiligten verleiht. Die Leichte Sprache steckt jedenfalls voller Tücken und Paradoxien, und "barrierefreie Kommunikation" beschränkt sich womöglich zwangsläufig auf den Austausch von Banalitäten.

Thomas Niehr konfrontiert in seinem Text die Traditionen der moralisch-politischen Sprachkritik und der kritischen Diskursanalyse mit einer programmatisch strikt deskriptiven Politolinguistik. Auch die Schwierigkeiten dieser Position (kann man einen "Euphemismus" konstatieren, ohne eine Position einzunehmen in der Frage, was das Bezeichnete "wirklich" ist?) werden eingehend diskutiert. Die geforderte engagierte Neutralität fachlicher Analysen kann freilich nicht verhindern, dass politische Akteure ihre Ergebnisse für die eigene Position vereinnahmen, und vielfach wird ja auch bereits das aufklärerische Freilegen latenter "Spielregeln" (etwa: Produktion und Pflege konsistenter Images) als politische Positionierung verstanden. Und gerade der Duktus der strikten Deskriptivität, der vollständigen wissenschaftlichen Neutralität, schafft nolens volens politische Autorität in einem Diskurs, in dem die Journale wimmeln von Statistiken, Infografiken, Tabellen, Zahlen, die allesamt eben das konnotieren: wissenschaftliche Neutralität. Wo allenthalben politische Entscheidungen gegenüber dem Publikum als Sachzwänge mit wissenschaftlicher (oder pseudowissenschaftlicher) Expertise unterfüttert werden (man denke nur an die "Wirtschaftsweisen"), ist Neutralität schwer durchzuhalten.

In einer demokratischen Szene ist "Charisma" eine Zuschreibung, die den mit ihr ausgezeichneten Akteur zugleich in dieser Szene verankert und über sie hinaushebt. Pamela Steen geht der Frage nach, welche Figuren, Deutungsmuster und Argumente bei der medialen Zuschreibung von "Charisma" eingesetzt werden. Im Sinne Kenneth Burkes "passt" ein charismatischer Akteur prima facie nicht in eine demokratische Szene (scene-agent-ratio). Also muss dramaturgisch entweder die Szene Eigenschaften haben, die nur mit Charisma zu bewältigen sind, oder das Charisma des Akteurs muss demokratisch eingebunden und gewissermaßen entschärft werden. Diese Ambivalenzen werden, wie Steen zeigt, nach klaren und rekonstruierbaren Mustern medial prozessiert.

Wolf-Andreas Lieberts Beitrag handelt von Selbstermächtigung und Degradierungszeremonien in der massenmedialen Kommunikation. Harold Garfinkels interaktiv-mikrosoziologisches Konzept der Degradierungszeremonie wird auf verschiedene Praktiken der massenmedialen symbolischen "Hinrichtung" prominenter oder auch ganz und gar unbekannter Diskursteilnehmer übertragen. Solche medialen shit storms sind einesteils leicht als MobVerhalten zu moralisieren, weil stets über einen Opferbonus verfügt, wer ihnen ausgesetzt ist. Anderenteils können sie auch taktisch provoziert und zur Selbststilisierung in einer einsamen, aber eben darum heldenhaften Außenseiterrolle genutzt werden. Zu der gehört es ja, dass man nichts gibt auf die Meinung der verblendeten Massen. Letzteres dürfte der Fall gewesen sein bei Sibylle Lewitscharoffs Rede zur Reproduktionsmedizin, die bis in die Einzelheiten einer bereits von Martin Walser und Botho Strauß erprobten Selbstskandalisierungsstrategie folgte. Es ist die massendemokratische Szene selbst, deren provozierte Selbstentlarvung als "Mob" den einsamen Helden umso mehr erhöht. 
"Demokratie" - zwischen Kampfbegriff und Nebelkerze

Mit den kommunikativen Praktiken der Etablierung eines politischen Ausnahmezustandes befasst sich Anna-Lena Dießelmann anhand einer bisher kaum untersuchten Textsorte: Den nach innen und außen gerichteten Kommunikationen der Polizeieinheiten, die im Jahr 2007 den G8-Gipfel in Heiligendamm gegen Demonstranten und Protestierer abzusichern hatten. Die Autorin untersucht die operativen sprachlichen Kodierungen im Einsatz dieser Polizeigruppen vor Ort: Wie teilen sie die Demonstranten in handlungsrelevante Gruppen ein? AnnaLena Dießelmann wendet explizit die von Kenneth Burke begründete Methode des dramatism auf das Material an, wodurch sich die spezifischen Facetten der sprachlichen Dramatisierung und Normalisierung erheben lassen: Die "friedlichen Demonstranten", allenthalben zum Zweck (purpose) der polizeilichen Maßnahmen erhoben, kommen bei den operativen Kategorien gar nicht vor, als Akteure (agents) erscheinen nur Bedrohungs- und Feindkategorien. Indem die Polizeikommunikation eine dramatisch denormalisierte Szene voller Bedrohungen entwirft, bahnt sie zugleich der Akzeptanz außergewöhnlicher (und nicht selten auch außerrechtlicher) Maßnahmen den Weg. Die Autorin versteht Normen, Normalitäten und Ausnahmesituationen als sozial und performativ erzeugt und rahmt die allgegenwärtige Krisen-, Denormalisierungs- und Ausnahmerhetorik als ein (durchaus strategisch verwendbares) Instrumentarium zur Verschiebung der demokratischen Normalitätsgrenzen nach unten.

In ihrer Gesamtheit stehen die Beiträge dieses Heftes für Facetten des Verhältnisses von Demokratie und Sprache. Sichtbar werden die Umrisse dessen, was Kenneth Burke (1969) als die "repräsentativen Anekdoten" des symbolischen Ordnungssystems mit Namen Demokratie bezeichnet haben würde: Da es sich bei "Demokratie" um einen szenischen Begriff handelt, gehört es zu dessen "Grammatik", dass alle fünf Elemente der Dramaturgie (act, scene, agent, agency, purpose) demokratisch sein sollen. Im Gegenzug gibt es aber natürlich auch "undemokratische" (oder als solche eingestufte) Handlungen, Verhältnisse, Akteure, Mittel und Ziele. "Normal" sind die demokratischen Verhältnisse, wenn alle fünf Größen konsonant zueinander passen. Demokratische Wahlen verkörpern dramaturgisch das Hochamt, das demokratische Wähler, Parteien, Verhältnisse und Ziele engführt und eine legitime Regierung hervorbringt. Die Grammatik demokratischer Normalität erlaubt es aber auch, Akteure, Mittel, Ziele usw. als "undemokratisch" anzuprangern und sie dann, je nach Lage, zu verbieten, politisch zu bekämpfen, ernst zu nehmen usw. In jedem Falle bewirkt eine undemokratische Größe in einer demokratischen Szene eine kognitive und emotionale Dissonanz, die bearbeitet werden muss. Die Diagnose "Postdemokratie" erlaubt es, die fortdauernde Geltung demokratischer Ideale ebenso zu postulieren wie die Gefährdung dieser Ideale durch dissonante Mittel oder Akteure, die unkontrollierte Machtressourcen akkumuliert haben. Geerdet ist das symbolische System Demokratie in den Erfahrungen und Erwartungen der nationalen Öffentlichkeiten. Massenhafte Wahlabstinenz unterminiert den Passus der repräsentativen Anekdote, der die Legitimität demokratischer Macht an die Zustimmung von Mehrheiten bindet. Was als offizielle Gefährdung demokratischer Normalität zustimmungspflichtig etabliert ist (wie der "internationale Terrorismus" oder der "islamische Staat"), das kann jederzeit eingesetzt werden, um die Absenkung demokratischer Normalitätsstandards für die Massen unvermeidlich erscheinen zu lassen. Immerhin erfahren wir inzwischen ein Level allgemeiner Kommunikationsüberwachung durch NSA und Geheimdienste als "normal", von dem die wahrlich rührige Staatssicherheit nur träumen konnte. Und dass wir Konzerne, Behörden und Versicherungen freiwillig und großzügig mit den Daten beliefern, die zu unserer Manipulation und Steuerung 
erforderlich sind, das kann man offenbar ebenso gut als Ausdruck demokratischer Verhältnisse wie als deren Gefährdung kodieren. Schließlich haben wir ja nichts zu verbergen!

Die "Grammatik" der Demokratie durchsichtig und verständlich zu machen, gehört jedenfalls zu den Aufgaben einer sich kritisch verstehenden Diskurswissenschaft ebenso wie zu denen einer strikt deskriptiven Politolinguistik. Hybris wäre es indessen, wenn die Sprach- und Kommunikationswissenschaftler glaubten, sie könnten das aus ihrer Profession heraus besser als andere Beobachter der Gesellschaft. Auch Schriftsteller betreiben bisweilen höchst kundig angewandte Diskursforschung. Michel Houellebecq lässt in seinem jüngsten Roman (Soumission) in Frankreich eine gemäßigt islamistische Regierung an die Macht gewählt werden, weil die Parteien der Mitte die Herrschaft des Front National um jeden Preis verhindern wollen. Wie sich die akademische Intelligenz durch die Freuden der Polygamie und die reichlich fließenden saudischen Petrodollars mit der neuen (natürlich demokratischen) Ordnung und der islamischen Religion versöhnen lässt, das wird am Beispiel eines Literaturwissenschaftlers geschildert, der an der Sorbonne lehrt und ironischerweise ein Spezialist für den im 19. Jahrhundert zum Katholizismus konvertierten Literaten Huysmans ist. Die ultrakonservative "abendländische" Rechte wittert als erste die Chance, mit Hilfe der Imame den europäischen Wertverfall aufzuhalten, den die verweltlichten und angepassten christlichen Religionen nicht mehr aufhalten können oder wollen. Kurz: Von den rhetorischen Extrapolationen der demokratischen Grammatik verstehen nicht nur die Diskurslinguisten etwas.

\section{$5 \quad$ Danksagung}

Für die finanzielle Unterstützung der Tagung "Sprache und Demokratie" im Oktober 2014 (Polzin 2015; Vogel 2014b), auf welche ein Teil der hier abgedruckten Beiträge zurückgehen, danken wir der Philosophischen Fakultät der Universität Siegen. Den Referentinnen und Referenten der Tagung danken wir ebenso, wie all denen, die Vortragsthemen eingereicht haben, sowie all denen, die durch ihre Diskussionsbeiträge zum Gelingen der Tagung beigetragen haben. Wir danken ebenfalls den Kolleginnen und Kollegen, die uns bei der Begutachtung der Beiträge unterstützt haben sowie der Herausgeberin Elke Hentschel für die Aufnahme dieses Heftes in die frei zugängliche Zeitschrift Linguistik Online. Für die sorgfältige Einrichtung der Texte danken wir schließlich Magnus Rook.

\section{Literatur}

Adler, Anja (2013): "Liquid Democracy als Social Software für Parteien". Forschungsjournal Soziale Bewegungen 2: 71-83.

Boutet, Josiane (2012): "Language Workers: Emblemic Figures of Late Capitalism". In: Duchêne, Alexandre/Heller, Monica (Hrsg.): Language in late capitalism: Pride and profit. New York: Routledge. (= Routledge Critical Studies in Multilingualism): 207-229.

Burke, Kenneth (1969): A grammar of motives. Berkeley/Los Angeles/London: University of California Press.

Burkhardt, Armin (Hrsg.) (2000): Sprache des deutschen Parlamentarismus: Studien zu 150 Jahren parlamentarischer Kommunikation. Wiesbaden: Westdeutscher Verlag. 
"Demokratie" - zwischen Kampfbegriff und Nebelkerze

Busch, Albert (2005): "Sprachschichten: Demokratischer Wortschatz zwischen Experten und Laien". In: Kilian, Jörg (Hrsg.): Sprache und Politik: Deutsch im demokratischen Staat. Mannheim, Dudenverlag: 141-160. (= Thema Deutsch 6).

Coleman, Stephen (2007): "E-democracy: the history and future of an idea". In: Mansell, Robin E. et al. (Hrsg.): The Oxford handbook of information and communication technologies. Oxford, Oxford University Press: 362-382.

darkbln (2011): "5 Jahre Liquid Democracy in Deutschland". LiquidFeedback. Interactive Democracy.

http://blog.liquidfeedback.org/2011/08/17/5-jahre-liquid-democracy-in-deutschland/ [18.04.2014].

Diekmannshenke, Hans-Joachim (2005): "Mitwirkung von allen? Demokratische Kommunikation im Chat". In: Kilian, Jörg (Hrsg.): Sprache und Politik: Deutsch im demokratischen Staat. Mannheim, Dudenverlag: 258-277. . (= Thema Deutsch 6).

Dörner, Andreas/Vogt, Ludgera (Hrsg.) (1995): Sprache des Parlaments und Semiotik der Demokratie: Studien zur politischen Kommunikation in der Moderne. Berlin/New York: de Gruyter. (= Sprache, Politik, Öffentlichkeit 6).

Fields, A. Belden/Narr, Wolff-Dieter (1992): "Human Rights as a Holistic Concept". Human Rights Quarterly 14/1: 1-20.

Goffman, Erving (1974): Frame analysis: An essay on the organization of experience: New York et al.: Harper/Row.

Grotlüschen, Anke/Riekmann, Wiebke (2011): leo. Level-One Studie. Literalität von Erwachsenen auf den unteren Kompetenzniveaus: Presseheft. http://blogs.epb.unihamburg.de/leo/files/2011/12/leo-Presseheft_15_12_2011.pdf [20.01.2014].

Holly, Werner (1993): "Fernsehen und Streitkultur". In: Janota, Johannes (Hrsg.): Kultureller Wandel und die Germanistik in der Bundesrepublik. Vorträge des Augsburger Germanistentages 1991: Bd. I: Vielfalt der kulturellen Systeme und Stile. Tübingen, Niemeyer: $57-$ 66.

Holly, Werner/Schwitalla, Johannes (1995): "Explosiv - der heiße Stuhl. Streitkultur im kommerziellen Fernsehn". In: Müller-Doohm, Stefan/Neumann-Braun, Klaus (Hrsg.): Kulturinszenierungen - Kultureffekte. Frankfurt a. M., Suhrkamp: 59-88.

Kämper, Heidrun (2009): "Demokratiegeschichte des 20. Jahrhunderts als Zäsurgeschichte das Beispiel der frühen Weimarer Republik: Ein transdisziplinäres Forschungsprojekt". Deutsche Sprache (ds) 4: 352-369.

Kämper, Heidrun (2012): Aspekte des Demokratiediskurses der späten 1960er Jahre: Konstellationen ; Kontexte ; Konzepte. 1. Auflage, Berlin: de Gruyter. (= Studia linguistica Germanica 107) www.degruyter.com/doi/book/10.1515/9783110263435 [02.09.2015].

Kämper, Heidrun (2013): Wörterbuch zum Demokratiediskurs 1967/68. Berlin: de Gruyter.

Kämper, Heidrun/Haslinger, Peter/Raithel, Thomas (Hrsg.) (2014): Demokratiegeschichte als Zäsurgeschichte: Diskurse der frühen Weimarer Republik. Berlin: de Gruyter. (= Diskursmuster-Discourse Patterns 5).

Kilian, Jörg (1997): Demokratische Sprache zwischen Tradition und Neuanfang: Am Beispiel des Grundrechte-Diskurses 1948-49. Tübingen: Niemeyer. (= Reihe germanistische Linguistik 186). http://site.ebrary.com/lib/alltitles/docDetail.action?docID=10591743 [02.09.2015]. 
Kilian, Jörg (2003). "'Demokratie' als Merkwort der Nachkriegszeit. Linguistische Begriffsgeschichte im Zeichen der kognitiven Semantik". In: Dutt, Carsten (Hrsg.): Herausforderungen der Begriffsgeschichte. Heidelberg, Winter: 105-132. (= Beiträge zur Philosophie Neue Folge).

Klein, Josef (2002). "Politische Kommunikation - Sprachwissenschaftliche Perspektiven". In: Jarren, Otfried/Sarcinelli, Ulrich/Saxer, Ulrich (Hrsg.): Politische Kommunikation in der demokratischen Gesellschaft: Ein Handbuch mit Lexikonteil. Opladen, Westdeutscher Verlag: 186-210.

Klein, Josef (2005): "'Grundwortschatz' der Demokratie". In: Kilian, Jörg (Hrsg.): Sprache und Politik: Deutsch im demokratischen Staat. Mannheim, Dudenverlag: 128-140. (= Thema Deutsch 6).

Knobloch, Clemens (2015): Wie man öffentlich über Inklusion spricht (und was man daraus schließen kann). www.nachdenkseiten.de/?p=24597 [29.05.2015].

Läzer, Rüdiger (2000): "Von der 'Demokratie der Straße' zum demokratischen Parlament: Politische Streitkultur in Verhandlungen am Runden Tisch". In: Burkhardt, Armin/Pape, Kornelia (Hrsg.): Sprache des deutschen Parlamentarismus. Opladen/Wiesbaden, Westdeutscher Verlag: 431-450.

Meyer, Thomas (2002): "Mediokratie - Auf dem Weg in eine andere Demokratie?". In: Politik und Zeitgeschichte 16: 7-14. www.bpb.de/system/files/pdf/MIMC25.pdf [05.09.2015].

Narr, Wolf-Dieter (2012): Trotzdem: Menschenrechte!: Versuch, uns und anderen nach nationalsozialistischer Herrschaft Menschenrechte zu erklären. Köln: Komitee für Grundrechte und Demokratie.

Neuland, Eva (2005): "Ist eine Spracherziehung zur Demokratie möglich? Ein Diskussionsbeitrag". In: Kilian, Jörg (Hrsg.): Sprache und Politik: Deutsch im demokratischen Staat. Mannheim, Dudenverlag: 74-84. (= Thema Deutsch 6).

Pape, Kornelia (2005): "Informelles Regierungshandeln im demokratischen Prozess. In Spitzengesprächen zum Konsens". In: Kilian, Jörg (Hrsg.): Sprache und Politik: Deutsch im demokratischen Staat. Mannheim, Dudenverlag: 114-127. (= Thema Deutsch 6).

Polzin, Martin (2015): "Sprache und Demokratie: Interdisziplinäre Tagung vom 2. bis 4. Oktober 2014 in Siegen". Zeitschrift für germanistische Linguistik 43/1: 144-153.

Rilling, Rainer (1998): "Marktvermittelt oder selbstorganisiert?: Zu den Strukturen von Ungleichheit im Netz". In: Leggewie, Claus/Maar, Christa (Hrsg.): Internet \& Politik: Von der Zuschauer- zur Beteiligungsdemokratie? Köln, Bollmann: 366-380.

Schwitalla, Johannes (2001): "Konflikte und Verfahren ihrer Bearbeitung". In: Brinker, Klaus et al. (Hrsg.): Text- und Gesprächslinguistik: Ein internationales Handbuch zeitgenössischer Forschung = Linguistics of text and conversation. 2. Bd. Berlin, de Gruyter: 13741382. (= Handbücher zur Sprach- und Kommunikationswissenschaft 16).

Schwitalla, Johannes/Keim, Inken (1989): "Soziale Stile des Miteinander-Sprechens: Beobachtungen zu Formen der Konfliktbearbeitung in zwei Frauengruppen". In: Hinnenkamp, Volker/Selting, Margret (Hrsg.): Stil und Stilisierung: Arbeiten zur Interpretativen Soziolinguistik. Tübingen, Niemeyer: 83-121.

Stefanowitsch, Anatol (2014): "Leichte Sprache, komplexe Wirklichkeit". (= Politik und Zeitgeschichte 9-11). Www.bpb.de/apuz/179343/leichte-sprache-komplexe-wirklichkeit [07.05.2015]. 
"Demokratie" - zwischen Kampfbegriff und Nebelkerze

Vogel, Friedemann (2014a): "Linguistik als Kampfsport. Auf der Suche nach Paradigmen demokratischen Sprechens in Alltag, Medien und Recht". Linguistik online 69/7: 91-108. doi: 10.13092/lo.69.1658.

Vogel, Friedemann (2014b): Sprache und Demokratie - Dokumentation einer Tagung. www.sud.friedemann-vogel.de [05.09.2015].

Vogel, Friedemann/Luth, Janine/Ptashnyk, Stefaniya (i. E.): "Konflikt und Konfliktbewältigung im Spiegel der Sprache, oder: Plädoyer für die Suche nach einem linguistischen Beitrag zur Befriedung Europas". In: Vogel, Friedemann/Luth, Janine/Ptashnyk, Stefaniya (Hrsg.): Linguistische Zugänge zu Konflikten in europäischen Sprachräumen. Korpus Pragmatik-kontrovers. Heidelberg: Winter.

Weingart, Peter (2001): Die Stunde der Wahrheit?: Zum Verhältnis der Wissenschaft zu Politik, Wirtschaft und Medien in der Wissensgesellschaft. Weilerswist: Velbrück. 